University of New Hampshire

University of New Hampshire Scholars' Repository

\title{
$4-1989$
}

\section{Chirp subbottom profiler for quantitative sediment analysis}

Steven G. Schock

Florida Atlantic University

Lester R. LeBlanc

Florida Atlantic University

Larry A. Mayer

University of New Hampshire, larry.mayer@unh.edu

Follow this and additional works at: https://scholars.unh.edu/ccom_affil

Part of the Geophysics and Seismology Commons, and the Oceanography and Atmospheric Sciences and Meteorology Commons

\section{Recommended Citation}

Steven G. Schock, Lester R. LeBlanc, and Larry A. Mayer (1989). "Chirp subbottom profiler for quantitative sediment analysis." GEOPHYSICS, 54(4), 445-450. doi: 10.1190/1.1442670

This Article is brought to you for free and open access by the Center for Coastal and Ocean Mapping at University of New Hampshire Scholars' Repository. It has been accepted for inclusion in Affiliate Scholarship by an authorized administrator of University of New Hampshire Scholars' Repository. For more information, please contact Scholarly.Communication@unh.edu. 


\title{
Chirp subbottom profiler for quantitative sediment analysis
}

\author{
Steven G. Schock*, Lester R. LeBlanc*, and Larry A. Mayer $\ddagger$
}

\begin{abstract}
A wide-band, frequency-modulated, subbottom profiling system (the chirp sonar) can remotely determine the acoustic attenuation of ocean sediments and produce artifact-free sediment profiles in real time. The chirp sonar is controlled by a minicomputer which performs analog-to-digital and digital-to-analog conversion, correlation processing, and attenuation estimation in real time. The minicomputer generates an FM pulse that is phase- and amplitude-compensated to correct for the sonar system response. Such precise waveform control helps suppress correlation noise and source ringing. The chirp sonar, which has an effective bandwidth of $5 \mathrm{kHz}$. can generate chirp (Klauder) wavelets with a tuning thickness (Rayleigh's criterion for resolution) of approximately $0.1 \mathrm{~ms}$. After each return is correlated, a computationally fast algorithm estimates the attenuation of subbottom reflections by waveform matching with a theoretically attenuated waveform. This algorithm obtains an attenuation estimate by minimizing the mean-square error between the autocorrelation function of the theoretically attenuated wavelet and the autocorrelation function of the subbottom reflection.

The chirp sonar was tested in Narragansett Bay, R.I. along a line that had been previously cored. Experimental results show that correlation noise from the seafloor reflection was below $-60 \mathrm{~dB}$, the quantization noise level, thereby allowing detection of small subbottom impedance contrasts and accurate estimation of attenuation. Attenuation coefficient estimates from this sandy region agree with in-situ measurements made by other investigators.
\end{abstract}

\section{INTRODUCTION}

Acoustic methods for remotely measuring marine sediment properties have a wide range of marine geologic and geotechnical applications. Before accurate quantitative measurements of subsurface properties can be realized, specialized sonar equipment and signal processing techniques must be developed. We describe a powerful research tool, the chirp sonar, a quantitative acoustic measurement system used to provide real-time, artifact-free, high-resolution seismograms and estimates of acoustic attenuation in marine sediments. The chirp sonar, which contains a wide-band swept FM source, was developed by researchers at the University of Rhode Island and Dalhousie University as part of a long-term research project to determine the feasibility of performing remote quantitative measurements of marine sediments with broadband acoustic sources.

The chirp sonar system is controlled by a MASSCOMP minicomputer and is constructed entirely of linear transmitting and receiving components that enable exact waveform control (Figure 1). The minicomputer performs analog-todigital (A/D) and digital-to-analog (D/A) conversion, correlates digitized returns, generates a correlogram, and estimates the attenuation of the chirp (Klauder) wavelets, all in real time. The D/A converter generates an amplitude- and phasecompensated frequency-modulated waveform which is the pilot signal for a $1.2 \mathrm{~kW}$ audio power amplifier. A transformer provides impedance matching for the transducer, a piezoceramic annular cylinder mounted in a conical baffle. The transducer and receiver array are mounted on a tow body. The receiver line array output is linearly amplified prior to $\mathrm{A} / \mathrm{D}$ conversion. The system can transmit usable energy from 2 to $20 \mathrm{kHz}$; its transfer function (magnitude) is plotted in Figure 2. For more details on the chirp sonar system refer to Schock et al. (1986) and Mayer and LeBlanc (1983).

We use the term "chirp sonar" to describe our highresolution. quantitative, subbottom-profiling system in order to distinguish this FM sonar from marine Vibroseis systems. The design criteria for the two systems are quite different. Marine Vibroseis systems are designed for deep penetration to detect hydrocarbon traps and to locate significant structural features and major stratigraphic horizons. Consequently, those seismic systems operate at frequencies below $500 \mathrm{~Hz}$ with bandwidths that rarely exceed $100 \mathrm{~Hz}$ (Broding et al., 1971: Clay and Liang, 1962; 1964). In contrast, the chirp

Presented in part at the 18th Annual Offshore Technology Conference. Manuscript received by the Editor November 20, 1987; revised manuscript received October 5,1988 .

* Department of Ocean Engineering. South Lab, University of Rhode Island, Narragansett, RI 02882.

$\Varangle$ Department of Oceanography, Dalhousie University, Halifax. Nova Scotia, Canada.

(1989 Society of Exploration Geophysicists. All rights reserved. 
sonar system is designed to measure ocean sediment properties while subbottom profiling. The required vertical resolution for those quantitative measurements necessitates signal bandwidths greater than $1 \mathrm{kHz}$.

The design goals for the chirp sonar are (1) a vertical resolution on the order of $10 \mathrm{~cm}$ and (2) real-time, artifact-free subbottom profiles and measurements of the sediment column attenuation to $100 \mathrm{~m}$. A resolution of $10 \mathrm{~cm}$ is chosen because in most marine environments bioturbation (burrowers cycling sea-floor sediments) mixes sediments over this depth (Mayer, 1979). Since sediment properties do not vary significantly over this interval, the acoustic response of the sediment column is adequately represented when wavelet resolution is $10 \mathrm{~cm}$ or better. This requirement is met if the tuning thickness (Rayleigh's criterion for resolution) of the chirp (Klauder) wavelet is about $0.1 \mathrm{~ms}$, where the exact value depends upon the compressional wave velocity of the sediments. Resolution of zerophase wavelets is discussed by Kallweit and Wood (1982).

Artifact-free subbottom records and attenuation estimates require reducing the amplitudes of water-path multiples and suppressing correlation and noncorrelation noise. Since the chirp sonar usually operates above $1 \mathrm{kHz}$, acoustic wavelengths are short enough to permit highly directional arrays. By mounting pressure release surfaces on the acoustic arrays, all water-column multiples can be practically eliminated, with the exception of the water-bottom multiple, which repeats at intervals of twice the water-column traveltime.

Noncorrelation noise includes acoustic, quantization, and electrical noise. These "system" noise sources are attenuated by correlation (matched-filter) processing. The signal-to-noise (S/N) improvement for matched filtering a known signal with inband white noise is given by

$$
\mathrm{S} / \mathrm{N}_{\text {out }}-\mathrm{S} / \mathrm{N}_{\text {in }}=10 \log _{10}(T B W) \mathrm{dB},
$$

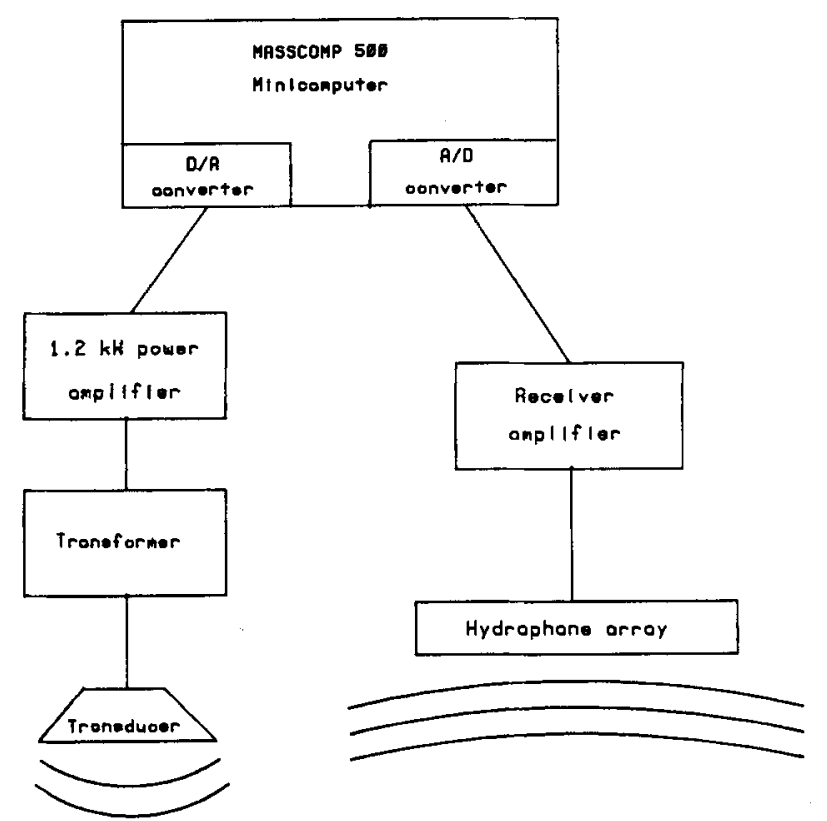

FIG. 1. Configuration of the chirp sonar system. where $T$ is the length and $B W$ is the bandwidth of the frequency-modulated pulse (Geyer, 1969). For more information on matched filters and noise suppression refer to Turin (1960), Krey (1969), and Klauder et al. (1960).

Correlation noise at the output of the matched filter can add a significant amount of inband noise to the entire return. The correlation side lobes of strong reflections can interfere with weaker reflections and prevent the detection of fine layering as well as the measurement of the sediment properties (Schock et al., 1986; Edelmann and Werner, 1982b). Correlation side lobes must be reduced to below the system noise level prior to utilizing spectral analysis techniques to estimate the attenuation of weak reflections. To ensure accurate spectral estimates of waveforms returned from small impedance contrasts, we require side-lobe levels below $-80 \mathrm{~dB}$. Correlation noise reduction methods are discussed in the Appendix.

The time interval available for real-time processing of a subbottom return cannot exceed the time that a data buffer is available; i.e., the period when the data acquisition control processor in the minicomputer is not using the buffer. With chirp pulse repetition rates of two transmissions per second, computationally intensive routines cannot be implemented on our portable data acquisition minicomputer. Consequently, we run two signal processing routines which efficiently perform matched-filter pulse compression and chirp wavelet attenuation estimation.

In the following discussions we describe our signal processing routines. These techniques are demonstrated by analyzing a subbottom profile acquired by the chirp sonar along a line of core sites in Narragansett Bay, R. I. The experiment produced a subbottom profile with the system noise level of $-63 \mathrm{~dB}$, the side-lobe levels below $-63 \mathrm{~dB}$, and attenuation coefficient estimates that agree with those published by other investigators for similar sediment types.

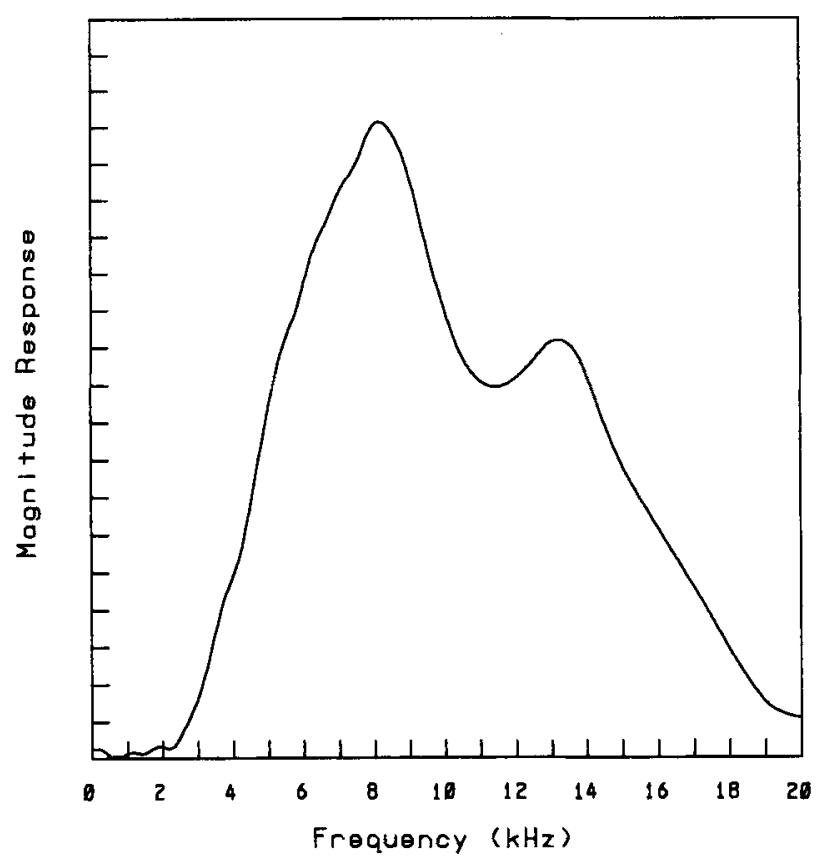

FIG. 2. Magnitude of the chirp sonar system transfer function. 


\section{ATTENUATION ESTIMATION}

The amplitude frequency response of an isotropic, lossy medium can be modeled by the equation,

$$
A(x, f)=A_{0} e^{-b(f) x}
$$

or, equivalently,

$$
A(x, f)=A_{0} 10^{-\alpha(f)\langle x / 20)},
$$

where

$$
\alpha(f)=k f^{n}=20 b(f) \log e .
$$

$A_{0}$ is a scaling constant; $x$ is the distance the wavelet traveled $(\mathrm{m}) ; b$ is the attenuation (nepers $/ \mathrm{m}) ; \alpha$ is at tenuation $(\mathrm{dB} / \mathrm{m}) ; k$ is the effective attenuation coefficient $(\mathrm{dB} / \mathrm{m} \mathrm{kHz})$; and $f$ is frequency $(\mathrm{kHz})$. The coefficient $k$, estimated from the acoustic data, is called the effective attenuation coefficient because it accounts for energy losses due to intrinsic absorption, reflection, refraction, and scattering (Hamilton, 1976). Assuming that energy damping is small, the relationship between attenuation and $Q$ is

$$
\frac{1}{Q}=\frac{b V}{\pi f},
$$

where $V$ is the wave velocity and $f$ is frequency $(\mathrm{Hz})$ (Hamilton, 1972; Jacobson, 1987).

Techniques for estimating attenuation from reflection profiles depend on pulse bandwidth and the assumption concerning the value of $n$ in equation (2). If the pulse is narrowband, measurements of the decay of the pulse envelope peak versus traveltime are sufficient to estimate the attenuation $b$ at the carrier frequency. If the pulse is wideband, attenuation is normally found by estimating the values for $k$ and $n$ in equation (2) by spectral ratios (Tullos and Reid, 1969; Jacobson et al., 1981), wavelet rise times (Jannsen et al., 1985), transfer functions (Raikes and White, 1984), spectral shifts (Kuc, 1984), spectral centroids (Fink et al., 1983), zero-crossing density (Flax et al., 1983), or wavelet modeling (Jannsen et al., 1985). Many investigators assume $n=1$ to simplify their attenuation estimation procedures because, in many cases, measurements show that $n$ is not statistically different from 1. Jacobson (1987) discusses the problems associated with assuming frequency-independent $Q$

The method we present for estimating attenuation is similar to time-domain wavelet modeling (Jannsen et al., 1985) which finds the attenuation by determining the best fit of synthetically attenuated wavelets to a seismogram reflection. The method we propose is also a time-domain solution, but attenuation is determined by finding the least-squares fit between the autocorrelation functions of the synthetically attenuated chirp wavelet and the time-gated subbottom reflection. The motivation behind this technique is our desire to estimate attenuation in the presence of scattering and interlayer interference while performing real-time data acquisition. We do not compare the accuracy of our wavelet modeling method to the accuracy of other attenuation estimation procedures; however, Jannsen et al. (1985) provide some comparisons.

Although our attenuation estimation technique can use any attenuation function, we use the function described by equa- tion (2). The attenuation function $a(t)$ has a power spectral density

$$
S_{a}(f)=S_{0} 10^{\left(-k f^{n / 10) x}\right.}=S_{0} 10^{-k^{\prime} f n / 10}
$$

where we call $k^{\prime}$ the attenuation rolloff $(\mathrm{dB} / \mathrm{kHz})$. If the matched-filter impulse response is $f(t)$, the autocorrelation function of the synthetic, normalized, attenuated wavelet $\hat{R}_{s}(t)$ is calculated in the following manner:

$$
\begin{aligned}
R_{f}(t) & =f(t) * f(-t) \\
R_{f a}(t) & =R_{f}(t) * a(-t) \\
R_{s}(t) & =R_{f a}(t) * R_{f a}(-t) \\
\hat{R}_{s}(t) & =\frac{\left[R_{s}(t)\right]}{\left[R_{s}(0)\right]},
\end{aligned}
$$

where the circumflex indicates a normalized function.

After assuming a value for $n$, the functions $\hat{R}_{s}(t)$ are generated for every value of attenuation rolloff $k^{\prime}$ expected for the sediment type and acoustic penetration (e.g., 200 curves ranging from 0 to $20 \mathrm{~dB} / \mathrm{kHz}$ ). Figure 3 is an example of five synthetic curves that are stored for the comparison. Note that the main lobe widens monotonically with greater attenuation rolloff.

A least-squares fit is performed between $\hat{R}_{s}(t)$ and $\hat{R}_{m}(t)$ where $\hat{R}_{m}(t)$, the measured, normalized autocorrelation function of the time-gated subbottom reflections, is given by

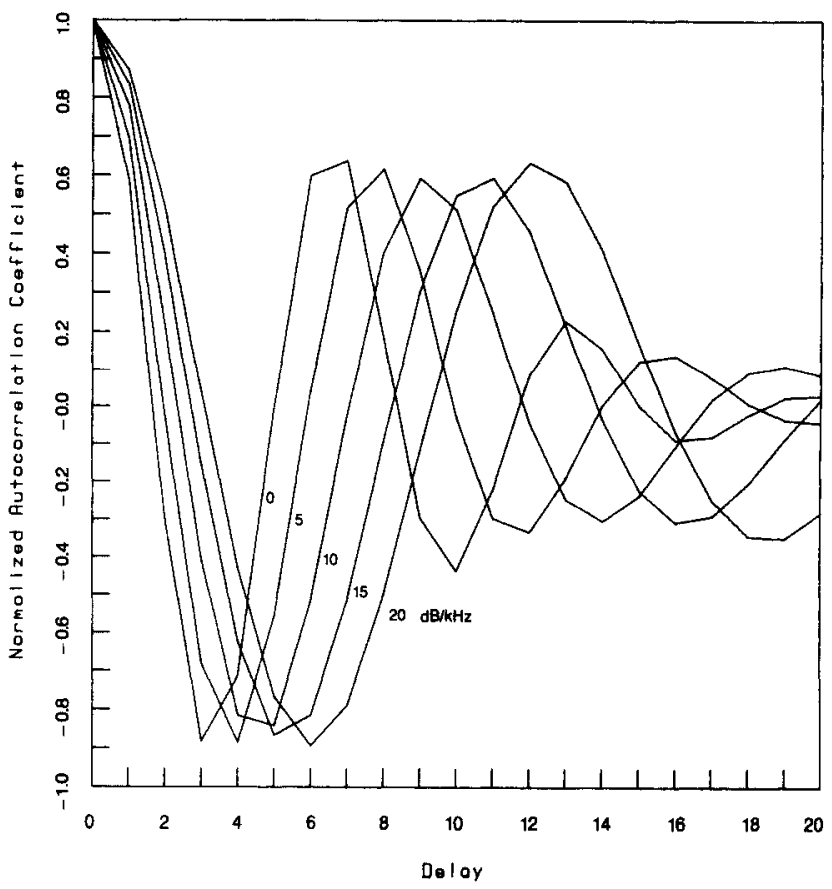

Fig. 3. Plot of five autocorrelation functions of chirp wavelets with attenuation rolloffs ranging from 0 to $20 \mathrm{~dB} / \mathrm{kHz}$. The autocorrelation function of a sediment interlayer reflection is fitted to one of these autocorrelated wavelets to determine the attenuation rolloff of the reflection. The sampling rate is 40 $\mathrm{kHz}$. 


$$
\dot{R}_{m}(t)=R_{y f}(t) * R_{y f}(-t)
$$

and

$$
\hat{R}_{m}(t)=\frac{\left[R_{m}(t)\right]}{\left[R_{m}(0)\right]},
$$

where $y(t)$ is the subbottom return and $R_{y f}(t)=y(t) * f(-t)$. The mean-square error $e$ between $\hat{R}_{s}(t)$ and $\hat{R}_{m}(t)$ is represented in discrete notation by

$$
e=\frac{1}{n^{\prime}} \sum_{n=0}^{n^{\prime}-1}\left\{\hat{R}_{m}(n)-\hat{R}_{s}(n)\right\}^{2}
$$

A search is made to find the stored function $\hat{R}_{s}(n)$ that yields $e_{\min }$, the minimum mean-square error. The attenuation rolloff $k^{\prime}$ that corresponds to best-fit curve $\hat{R}_{s}(n)$ is divided by distance $x$ to obtain $k(\mathrm{~dB} / \mathrm{m} \mathrm{kHz})$, the effective attenuation coefficient for the upper $x / 2$ meters of sediment. The attenuation coefficient estimates for a subbottom layer are calculated by dividing the difference between the upper and lower layer interface attenuation rolloffs by twice the layer thickness. A threshold for $e_{\min }$ is picked based on the $S / N$ at the output of the matched filter and the degrees of scattering and layering interference. If $e_{\min }$ is above the selected threshold, the estimate is rejected.

Time-domain attenuation estimation has some nice features: In practice, the mean-square error calculation uses only the first few delays of the autocorrelation function main lobe. These first few delays contain sufficient spectral information to estimate the attenuation rolloff of the gated reflection. The tail of the autocorrelation function, which contains most of the delayed scattering events, is ignored. Therefore, problems associated with strong, closely spaced reflections and scatterers that appear outside the main lobe are reduced. Additionally, synthetic subbottom models show that lateral averaging of attenuation coefficients for a given time window can further improve the estimate if there is any variability in separation of the closely spaced reflections.

Another important strength of our time-domain approach, demonstrated by synthetic subbottom modeling, is that the variance of the attenuation estimate is not very sensitive to the number of delays used in the mean-square error calculation [equation (7)]. In fact, we have found there is little benefit to using more than four delays (the delay interval is based on the Nyquist sampling rate) when searching for $e_{\min }$. Since only $p$ delays are needed, the number of summations $n^{\prime}$ in equation (7) can be reduced to $p$. This allows us to reduce the number of delays needed to calculate the measured autocorrelation function $R_{m}(t)$, which can be expressed (in discrete form) as

$$
R_{m}(n)=\sum_{j=0}^{\ell-1} R_{y f}(j) R_{y f}(j+n), \quad n=0,1, \ldots, p,
$$

where $\ell$ equals the number of samples in the sliding time window. Recall that the value of the minimum square error threshold determines the acceptability of the attenuation estimate for every window position. If the estimate is not rejected when the square error threshold is set low and the number of

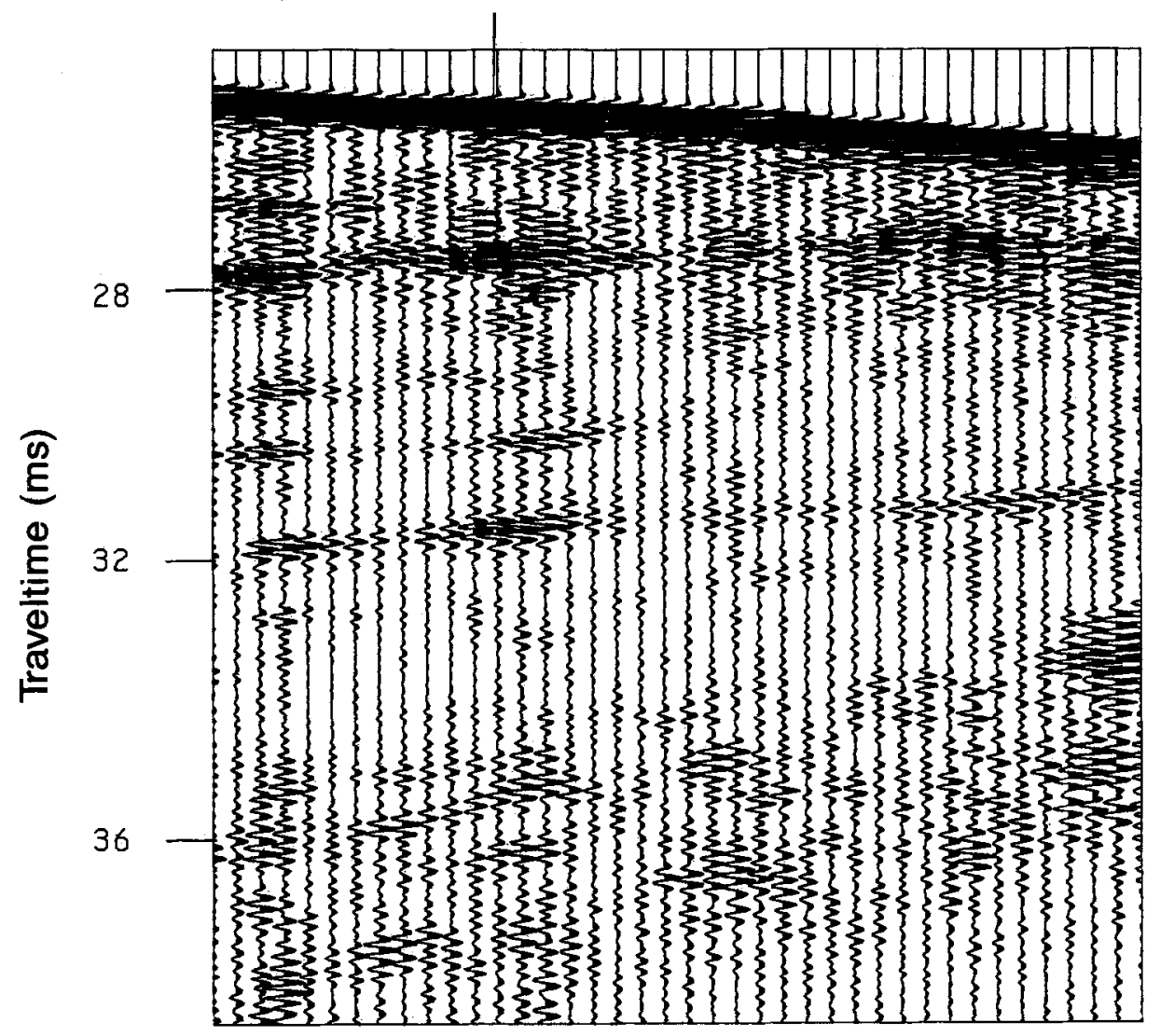

FIG. 4. Section from a chirp subbottom profile of the west passage of Narragansett Bay, R.I. Water depth is approximately $20 \mathrm{~m}$. At the sediment-water interface, the reflection of each return overlaps four adjacent returns. The rms noise level, measured before the sediment-water interface, is $-63 \mathrm{~dB}$. The deepest reflection horizon corresponds to the sand-bedrock boundary. 
delays is set high, the main lobes of the two autocorrelation functions will fit closely, thereby providing the best attenuation estimates. A tradeoff exists between the accuracy of the attenuation estimate and the computational speed of the algorithm. These simplified algorithms provide an efficient method of estimating the attenuation coefficient $k$, thereby permitting the calculation of real-time vertical profiles of attenuation coefficient estimates.

\section{CHIRP SONAR EXPERIMENT}

A subbottom profile was acquired along a line of coring sites at the proposed location of the Jamestown Bridge in Narragansett Bay, R. I. The matched-filter impulse response was an $8 \mathrm{~ms}, 2.5-12 \mathrm{kHz}$ linear FM sweep. The amplitude spectrum of the chirp wavelet was identical to the minimum four-sample Blackman-Harris window. The desired far-field waveform was generated by a slightly nonlinear pilot sweep which compensated for the phase and amplitude spectra of the sonar system's transfer function (as described in the Appendix.) Figure 4 contains the subbottom profile. The seismogram noise level, gated just before the first reflection, is $63 \mathrm{~dB}$ down from the sediment-water interface reflection. No side lobes are visible in the seismogram. Note that the wide dynamic range of the acoustic record allows the detection of weak layering. Core records show that the sediment structure consists of a

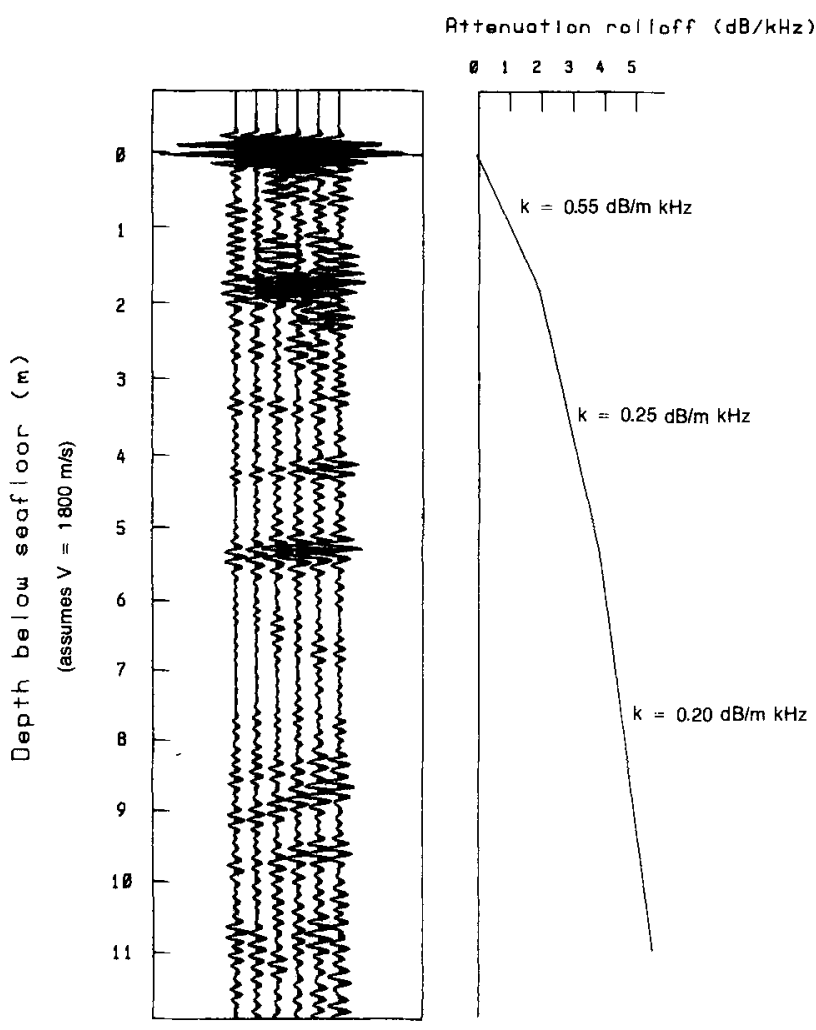

Fig, 5. Plot of the averaged attenuation rolloff versus depth The attenuation rolloff, measured at the three strongest inter. layer reflections, is laterally averaged over the six returns plotted to the left. The effective attenuation coefficient $k$ is calculated by dividing the difference between the attenuation rolloffs across a sediment layer by twice the layer thickness. wedge of sands and silts over bedrock. In Figure 5 attenuation rolloff is plotted against depth below the sea floor, and the effective attenuation coefficients are given for three sediment layers. A sound speed of $1800 \mathrm{~m} / \mathrm{s}$, a typical value for sands (Hamilton, 1972), was used to calculate the depth of the gated reflections. The attenuation coefficient estimates agree with in-situ measurements of similar sediments which are summarized in Hamilton (1976). The decrease in the effective attenuation coefficients with depth supports the results of Hamilton (1976).

\section{CONCLUSIONS}

The use of high-resolution seismic systems for the remote classification of marine sediments depends on the development of profiling systems that have high resolution, a wide dynamic range, substantial subbottom penetration, and the ability to generate quantitative estimates of sediment properties. We have approached this problem through the use of a computercontrolled, towed. sonar system (the chirp sonar) that uses a slightly nonlinear pilot sweep that is phase- and amplitudecompensated and matched-filter processing to produce highresolution seismograms which contain no visible ringing or side lobes. Tests of our system in Narragansett Bay, R.I. have demonstrated the ability to detect weak layering and to generate signal-to-noise ratios of more than $60 \mathrm{~dB}$. The wide dynamic range is due, in part, to the extreme care taken in designing the sonar equipment and the pilot signal which compensates for the entire system transfer function and minimizes the disrupting effects of side lobes. With side lobes and source ringing suppressed, real-time attenuation estimates are realizable. We presented a robust time-domain algorithm that can quickly estimate attenuation by waveform matching. Attenuation estimates, calculated using our Narragansett Bay data set, agree with attenuation coefficients derived from insitu sediment attenuation measurements performed by other investigators for similar sediments.

The chirp sonar, with its broad bandwidth and precise waveform control, can produce high-resolution, high $\mathrm{S} / \mathrm{N}$ subbottom reflection profiles, vertically and horizontally continuous attenuation coefficient sediment profiles, and, if desired, sediment reflectivity measurements. Such capabilities make the chirp sonar a well-suited instrument for remote quantitative sediment studies.

\section{ACKNOWLEDGMENTS}

This research was supported by the Office of Naval Research under contract no. N00014-81-C00062 to the University of Rhode Island and contract no. N00014-83-G-0114 to Dalhousie University. The MASSCOMP 500 minicomputer was provided under a DoD Research Initiative Grant.

\section{REFERENCES}

Broding, R. A., Hess, J. M., and Wanous, R. E., 1971, A high-power computer-controlled marine Vibroseis system: Inst. Electr. Electron. Eng., Trans. Geosci. Electron., GE-9, 90-95.

Clay, C. S., and Liang, W. L., 1962, Continuous seismic profiling with matched filter detector: Geophysics, 27, 786-795.

- 1964, Seisnic profiling with a hydroacoustic transducer and correlation receiver: J. Geophys. Res., 69, 3419-3428.

Cunningham, A. B., 1979, Some alternate vibrator signals: Geophysics, 44, 1901-1921. 
Edelmann, H. A. K., and Werner, H., 1982a, The encoded sweep technique for Vibroseis: Geophysics, 47, 809-818.

$1982 \mathrm{~b}$, Combined sweep signals for correlation noise suppression: Geophys. Prosp., 30, 786-812.

Fink, M., Hottier, F., and Cardoso, J. F., 1983, Ultrasonic signal processing for in vivo attenuation measurement: short time Fourier analysis: Ultrasonic Imaging, 5, 117-135.

Flax, S. W., Pelc, N. J., Glover, G. H., Gutmann, F. D., and McLachlan, M., 1983, Spectral characterization and attenuation measurements in ultrasound: Ultasonic Imaging, 5, 95-116.

Geyer, R. L., 1969, The Vibroseis system of seismic mapping: J. Can. Soc. Explor. Geophys., 6, 39-57.

Goupillaud, P. L., 1976, Signal design in the "Vibroseis" technique: Geophysics, 41, 1291-1304.

Hamilton, E. L., 1972, Compressional-wave attenuation in marine sediments: Geophysics, 37, 620-646.

1976 , Sound attenuation as a function of depth in the sea floor: J. Acoust. Soc. Am., 59, 528-535.

Harris, F. J., 1978, On the use of windows for harmonic analysis with the discrete Fourier transform: Proc., Inst. Electr. Electron. Eng., 66, 51-83.

Jacobson, R. S., Shor, G. G., and Dorman, L. M., 1981, Linear inversion of body wave data--Part II: attenuation versus depth using spectral ratios: Geophysics, 46, 152-162.

1987, An investigation into the fundamental relationships between attenuation, phase dispersion, and frequency using seismic refraction profiles over sedimentary structures: Geophysics, 52, $72-87$.

Jannsen, D., Voss, J., and Theilen, F., 1985, Comparison of methods to determine $Q$ in shallow marine sediments from vertical reflection seismograms: Geophys. Prosp., 33, 479-497.
Kallweit, R. S., and Wood, L. C., 1982, The limits of resolution of zero-phase wavelets: Geophysics, 47, 1035-1046.

Klauder, J. R., Price, A. C., Darlington, S., and Albersheim, W. J., 1960, The theory and design of chirp radars: The Bell Sys. Tech. J., $39,745-808$.

Krey, T. H., 1969, Remarks on the signal to noise ratio in the Vibroseis system: Geophys. Prosp., 17, 206-218.

Kuc, R., 1984, Estimating acoustic attenuation from reflected ultrasound signals: comparison of spectral-shift and spectral-difference approaches: Inst. Electr. Electron. Eng., Trans. Acoust., Speech, and Signal Proc., ASSP-32, 1-6.

Mayer, L. A., 1979, The origin of fine-scale acoustic stratigraphy in deep-sea carbonates: J. Geophys. Res., 84, 6177-6184.

Mayer, L. A., and LeBlanc, L. R., 1983, The chirp sonar: a new quantitative high-resolution profiling system, in Pace, N. G., Ed., Acoustics and the sea bed: Bath Univ. Press.

Raikes, S. A., and White, R. E., 1984, Measurements of earth attenuation from downhole and surface seismic recordings: Geophys. Prosp., 32, 892-919.

Rietsch, E., 1977, Vibroseis signals with prescribed power spectrum: Geophys. Prosp., 25, 613-620.

Schock, S. G., LeBlanc, L. R., and Mayer, L. A., 1986, Sediment classification using a wideband, frequency-modulated sonar system: Proc., 18th Ann. Offshore Tech. Conf., 389-398.

Tullos, F. N., and Reid, A. C., 1969, Seismic attenuation of Gulf Coast sediments: Geophysics, 34, 516-528.

Turin, G. L., 1960, An introduction to matched filters: Inst. Radio Eng., Trans. Inf. Theory, IT-6, 311-329.

Werner, H., and Krey, T., 1979, Combisweep-a contribution to sweep techniques: Geophys. Prosp., 27, 78-105.

\section{APPENDIX A}

\section{CORRELATION NOISE SUPPRESSION}

Not all correlation noise reduction methods are appropriate for quantitative subbottom profiling; many of these methods do not meet our requirement that correlation side-lobe levels be at least $80 \mathrm{~dB}$ below the peak amplitude of the sea-floor reflection.

Correlation noise reduction techniques such as encoded sweeps, Combisweep (a trademark of Prakla-Seimos GmbH), and pseudorandom-coded signals are difficult to adapt to our application. Encoded sweeps achieve complete noise suppression at one code length from the center of a correlated reflection (Edelmann and Werner, 1982b); however, we require side lobes to be below the system noise floor immediately adjacent to a strong reflection. Combisweep uses several untapered linear sweeps of various bandwidths to shape the spectrum of the Vibroseis wavelet (Werner and Krey, 1979). Optimizing the Combisweep parameters to suppress side lobes below -80 $\mathrm{dB}$ is difficult with untapered sweeps. The pseudorandomcoded method of correlation noise suppression (Cunningham, 1979) is difficult to implement in a band-limited system because the pilot signal is essentially a continuous sine wave convolved with a random sequence of step functions with opposite polarities. It follows that the vibrator transmission includes a sequence of step responses which, when convolved with the reference coded signal, produces a correlogram with source ringing. Edelmann and Werner (1982a) state that no alternative signals have proved superior to the sweep signal.

Other correlation noise reduction techniques include nonlinear sweeps (Goupillaud, 1976; Rietsch, 1977) and sweep tapering (Klauder et al., 1960; Harris, 1978). A nonlinear sweep with end tapering or a tapered linear sweep can be selected to shape the chirp wavelet amplitude spectrum to meet the requirement that wavelet side-lobe levels quickly drop below $-80 \mathrm{~dB}$. The nonlinear sweep with end tapering is the preferred waveform because it utilizes the full dynamic range of the transmitting amplifier, thereby providing higher source levels at some frequencies. However, for this investigation a tapered linear sweep was selected, because (1) maximizing acoustic penetration is not critical for demonstrating our methods and (2) the complex spectrum of the tapered linear sweep is easily calculated. In our experiment, the amplitude spectrum of the chirp wavelet is set equal to the minimum four-sample Blackman-Harris window so that the wavelet has side-lobe levels below $-92 \mathrm{~dB}$ (Harris, 1978). The window function for an $N$ sample DFT is

$$
\begin{aligned}
w(n)= & 0.35875-0.48829 \cos \left(\frac{2 \pi}{N} n\right)+0.14128 \cos \left(\frac{2 \pi}{N} 2 n\right) \\
& -0.01168 \cos \left(\frac{2 \pi}{N} 3 n\right), \quad n=0,1, \ldots, N-1 .
\end{aligned}
$$

After specifying the phase function defining the linear sweep and the amplitude spectrum of the chirp wavelet, the parameters of the pilot sweep are easily calculated. Since the bottom response is unknown, the matched filter is designed to detect the unattenuated linear sweep. It follows that the magnitude response of the matched filter is the square root of the chirp wavelet amplitude spectrum. To fully utilize the system dynamic range and bandwidth, the magnitude responses of the system and matched filter should fit closely. The pilot signal is determined by deconvolving the far-field system impulse response from the matched-filter impulse response (Schock et al., 1986). As a result, the pilot signal is a slightly nonlinear sweep that provides phase compensation to eliminate source ringing and amplitude compensation to suppress correlation noise. 